\title{
PROMOÇÃO DA SAÚDE, CAPACIDADE FUNCIONAL E FUNÇÃO COGNITIVA EM IDOSOS: NOTAS DE UM ENTRELACE
}

\author{
HEALTH PROMOTION, FUNCTIONAL CAPACITY AND COGNITIVE FUNCTION \\ IN ELDERLY: NOTES FROM A ENTRELACE
}

\author{
Noemi Pereira dos Santos ${ }^{1}$ \\ Paulo Henrique Fernandes Ferreira ${ }^{2}$ \\ Mirian Dias Moreira e Silva ${ }^{3}$ \\ Mônica Karla Vojta Miranda ${ }^{4}$ \\ Anselmo Cordeiro de Souza ${ }^{5}$
}

RESUMO: Obetivou-se apontar breves notas introdutórias na literatura científica de
contribuições teóricas e práticas em promoção da saúde e possíveis entrelaçamentos com a
capacidade funcional e a função cognitiva de idosos. Trata-se de uma comunicação curta que
a partir do espaço cientifico e acadêmico, toma como estratégia a narrativa bibliográfica.
Tomando como critério de seleção os títulos de interesse e conveniência a partir da
combinação dos Descritores em Ciências da Saúde relacionados a temática. Utilizaram-se
as fontes de informação: SciELO, PubMed e Google Scholar, no período de Janeiro a Junho
de 2or8. Evidenciou-se o estilo de vida promotor de saúde e suas relações com a capacidade
funcional e função cognitivas em idosos, bem como suas intersecoes interdisciplinares
podem possibilitar novas alternativas ou caminhos para fazer frente as demandas
provocadas pelas recentes mas inexoráveis transições sociais, demográficas e
epidemilogicas. Concluimos que embora esse trabalho não seja uma ampla revisão da
literatura, traz dados importantes ao se propor o estudo interdisciplinar da promoção da
saúde relacionada a capacidade funcional e função cognitiva. Explicitando o entrelace de

I ORCID: https://orcid.org/oooo-0oo2-5090-9752. Fisioterapeuta pelo Centro Universitário Adventista de São Paulo (UNASP), Especialista em Fisioterapia Desportiva e Ortopédica, e em Fisioterapia em Geriatria pela Faculdade Venda Nova do Imigrante (FAVENI).E-mail:noemisantosi6@hotmail.com

${ }^{2}$ ORCID: https://orcid.org/oooo-0oo3-3983-955ı. Profissional de Educação Física pelo Centro Universitário Adventista de São Paulo, Pós- graduado em Fisiologia aplicada à reabilitação pela Faculdade Adventista da Bahia (FADBA).E-mail: paulo.henriqueff@hotmail.com

3 ORCID: https://orcid.org/oooo-ooor-7757-6129. Doutoranda em Ciências Médicas pelo Centro Universitário Saúde ABC (CUSABC), Mestre em Promoção da Saúde pelo Centro Universitário Adventista de São Paulo (UNASP), Fisioterapeuta pela UniverCidade e Médica pela Faculdade de Ciências Médicas e da Saúde de Juiz de Fora (FCMS/JF).E-mail:midiasjf@yahoo.com.br

4 ORCID: https://orcid.org/oooo-ooor-96ı-o468. Mestra em Promoção da Saúde pelo Centro Adventista de São Paulo. Mestre em Gestão de Empresas/Saúde pela Universidade Lusófona de Humanidades e Tecnologias. Docente na Universidade do Estado do Pará (UEPA).

s ORCID: http://orcid.org/oooo-ooo2-0156-716X. Mestre em Promoção da Saúde e MBA em Gestão de Pessoas por Competências pelo Centro Adventista de São Paulo (UNASP), Discente da Licenciatura em Educação Física pela UnifAVENI, Pedagogo pela Faculdade Paulista São José (FPSJ), Teólogo pelo Seminário Adventista Latino Americano de Teologia (SALT/FADBA). E-mail: anselmo.vivamelhor@hotmail.com. 
espaços, situações e mesmo saberes, num rico, promissor e frutífero dialogo a repercutir de modo positivo na qualidade de vida da pessoa idosa.

Palavras-chave: Capacidade Funcional. Cognição. Idoso. Promoção da Saúde.

ABSTRACT: The aim was to point out brief introductory notes in the scientific literature on theoretical and practical contributions to health promotion and possible interrelationships with the functional capacity and cognitive function of the elderly. It is a short communication that, from the scientific and academic space, uses bibliographic narrative as a strategy. Taking as a selection criterion the titles of interest and convenience based on the combination of Health Sciences Descriptors related to the theme. The following sources of information were used: SciELO, PubMed and Google Scholar, from January to June 20r8. The health-promoting lifestyle and its relationship with the functional capacity and cognitive function in the elderly, as well as their interdisciplinary intersections can enable new alternatives or ways to face the demands caused by the recent but inexorable social, demographic and epidemiological transitions. We conclude that although this work is not a comprehensive review of the literature, it brings important data when proposing an interdisciplinary study of health promotion related to functional capacity and cognitive function. Explaining the interweaving of spaces, situations and even knowledge, in a rich, promising and fruitful dialogue that has a positive impact on the quality of life of the elderly.

Key-Words: Functional capacity. Cognition. Aged. Health promotion.

\section{INTRODUÇÃO}

As caracteristicas demográficas mundiais mudaram condiseravelmente nas últimas décadas. Com projeções para um mundo com população prevalente de idosos com potencial impactante para o cenário global e nacional, reestruturando inclusive as politicas e investimentos em importantes segmentos da sociedade. Entre as reestruturações está a saúde que tem relevância capital para essa população, pois é comum ao processo de senescência uma serie de caracteristicas próprias da velhice, aumentado nesta fase por exemplo, a susceptibilidade a doenças crônicas (IBGE, 2010, NARSI 2008, ABDALA, 20I4, 20II).

Destacamos, que modo acertado, é mencionado que chegar a uma idade avançada no Brasil deixou de ser uma raridade, passando a ser uma realidade claramente presente. $O$ que tem suscitado estudos a respeito da temática previamente nos países desenvolvidos onde esse processo de envelhecimento começa primeiro, e depois nos países subdesenvolvidos. 
Estas pesquisas de diferentes abordagens na saúde do idoso tem como objetivo comum um melhor entendimento bem como promoção a uma velhice bem sucedida (LIMA, SILVA, GALHARDONI, 2008; KALACHE, 2008; NASRI, 2008).

Logo objetivamos por meio desta comunicação breve apontar breves notas introdutórias na literatura científica de contribuições teóricas e práticas em promoção da saúde e possíveis entrelaçamentos com a capacidade funcional e a função cognitiva de idosos.

\section{METODOLOGIA}

Nesta pesquisa e contribuição introdutória, nos limitamos à descrição de algumas abordagens e produções acadêmicas e cientificas das possíveis interseções entre um estilo de vida promotor da saúde e a função cognitiva em idosos, utilizando como estratégia a narrativa bibliográfica. Tomando como critério de seleção os títulos de interesse e conveniência a partir da combinação dos Descritores em Ciências da Saúde - DeCS: Promoção da Saúde, Estilo de vida, Cognição, idoso; dos Medical Subject Headings - MeSH Terms: Promoting Health, Lifestyle, Cognition, Aged; combinados a experessões em português

e inglês: Função Cognitiva e Capacidade Funcional dado serem termos não controlado recorrentes na literatura em relação a temática tanto nacional como internacionalmente. Utilizaram-se assim as fontes de informação: SciELO, PubMed e Google Scholar, no período de Janeiro a Junho de 2018.

\section{RESULTADOS E DISCUSSÃO}

A capacidade funcional (CF) pode ser definida como a eficiência do indivíduo em corresponder às demandas físicas do cotidiano, comtemplando desde atividades básicas para uma vida funcional independente (atividades da vida diária) até ações mais complexas da rotina diária (atividades instrumentais da vida diária) (CAMARA et al., 2008).

Dentre as dimensões para uma melhor qualidade de vida de idosos repercutindo diretamente em sua capacidade funcional está a função cognitiva, naturalmente afetada pela idade avançada, mas não necessesariamente disfuncional (LIRA, SANTOS, 2012; RABELO, 2009; RABELO, NERI 2005). Função cognitiva pode ser definida como o processo 
intelectual pelo qual uma pessoa toma conhecimento das ideias, percebe-as e compreende-as. Envolve todos os aspectos da percepção, pensamento, raciocínio e memória (CHODZKO-ZAJKO, MOORE, 1994).

- Apesar de a resposta cognitiva parecer se dar mais lenta entre os idosos, não significa a idade um fator impedidor para uma boa e otimizada função cognitiva. Neste sentido importa estímulos senso-motores capazes de despertar e exercitar as capacidades mentais associadas a cognição (LIRA, SANTOS, 2012; RABELO, 2009; RABELO, NERI 2005).

Para Figueiredo (2007), o estilo de vida é um importante incador de saúde cognitiva do idoso. Em sua revisão da literatura gerontológica que objetivou explorar o tema do envelhecimento saudável, deu-se destaque aos fatores contribuintes para a manutenção da qualidade de vida. Dentre os fatores pesquisados, o estilo de vida é considerado como um importante promotor de estímulos sócioemocionais que otimizam o funcionamento cognitivo.

Em face da realidade da transição demográfica e epidemiológica atual, se faz necessário uma atenção diferenciada ao fomento de mecanismos não apenas de controle e prevenção de doenças, mas estratégias eficazes para estabelecer de modo consistente a educação e a promoção da saúde da população idosa no cenário nacional e local. Ao subsidiar uma senescência ativa e bem sucedida, os fatores insalubres serão minimizados ou extintos, gerando um contexto familiar comunitário e social favorável a saúde do idoso em sua integralidade (KALACHE, 2008; VERAS, 2009; VERAS, 2008).

Lira e santos (2012) em seu artigo sobre a doença de Alzheimer trabalha da importante correlação entre função cognitiva e capacidade funcional. Apresentando que apesar de divergências entre estudos posteriores, há vários indicadores que apontam para relação linear entre função cognitiva e capacidade funcional. Ou seja quanto maior disfunção cognitiva menor capacidade funcional.

Segundo Alves, Leite e Machado (2008) na revisão do conceito e mensuração de incapacidade funcional aborda definições de incapacidade funcional compreendendo as dimensões físicas, cognitivas e emocionais, não obstante haja um predomínio de estudos que 
utilizem apenas as medidas físicas e instrumentais. Concluindo também que não há consenso quanto ao conceito e método de mensuração, ressalta porem que ainda diversos não são conflitantes.

Diante as divergências conceituais, operacionais, e mesmo em resultados de estudos de vários modelos metodológicos, parece ser de entendimento consolidado que tanto a função cognitiva, como a capacidade funcional influenciam e são influenciados pelo estilo de vida tanto no âmbito individual como coletivo. Se tornando o estilo e hábitos de vida importantes indicadores da qualidade de vida da população idosa.

O Estilo de Vida tem sido classicamente definico como conjunto de hábitos e costumes que são influenciados, modificados, encorajados ou inibidos pelo processo de socialização ao longo da vida. Esses hábitos e costumes incluem o uso de substâncias tais como o álcool, tabaco, chá ou café, hábitos dietéticos e de exercício. Eles têm importantes implicações para a saúde e são objeto de investigações epidemiológicas (MIRANDA, PORTO, SOUZA, 2018).

Ao relatar sobre um estudo de base populacional Valle et al.(2009) numa pesquisa mediada pelo uso do Mini Exame do Estado Mental - MEEM entre idosos de baixa escolaridade, encontra entre outras relações estudadas, significativa associação de hábitos de vida com a função cognitiva. Onde o menor consumo de frutas e hortaliças e pior desempenho no MEEM aponta para uma realidade preocupante. Sendo sugerido no estudo a necessidade de intervenções visando um aumento na ingestão destes alimentos.

De igual modo Antunes et al (2006) aborda do hábito de se exercitar apresentado-o como de importante impacto tanto na função cardiorrespiratória como na função cognitiva. Destacando entre os segmentos sociais com menor engagamento na pratica de exercícios físicos os indivíduos idosos. Representando tal realidade uma ameaça ao organismo humano, estimulando o aparecimento de doenças crônico-degenerativas. Sugere-se benefícios cognitivos provenientes de um estilo de vida ativo, como da pratica cumulativa durante toda a vida resultando assim uma "reserva cognitiva". Avaliando ainda o exercio físico como alternativa diferenciada para melhorar a função cognitiva, especialmente por sua aplicabilidade, por se tratar de um método economicamente acessível. 
Efetivamente o estilo de vida promotor da saúde pode ser um mediador importante na população idosa com hipertensão arterial po exemplo, por ser um segmento social mais propenso ao desenvolvimento da doença, sendo esta a principal doença crônica nesta população. As intervenções de estilo de vida podem ter ótima aderência, desde que bem orientadas especialmente através de um olhar contextualizado conduzido por uma equipe multiprofissional. (DIAS et al, 20II; BRASIL, 2006; TEIXEIRA et al. 2006; MIRANDA et al. 2002)

É apartir da interseção de diferentes olhares que efetivamente poderá se apreender melhor a realidade, efetivando-se em uma abordagem verdadeiramente contextualizada via a transversalização e interdisciplinaridade. Desenvolvendo-se um espaço propicio a promoção da saúde, criado do dialogo entre diversos saberes e interação multiprofissional. Percebendo como fator comum a efetividade de uma velhice bem sucedida preservando-se o quanto possível a função cognitiva, mediada pelo estilo de vida saudável, a interdisciplinaridade. (VITORINO, MIRANDA, WINTTER, 20I2; BRASIL 2006; OLIVEIRA, 2005)

Essencialmente a abordagem em promoção da saúde tem esse caráter interdisciplinar, transcendendo o paradigma centrado na doença ou prevenção, extrapolando em direção a um foco na saúde e em sua promoção. É esse entrelace entre as várias áreas do conhecimento que fundamenta esse campo/espaço em construção conhecido por promoção da saúde. $O$ que é natural dada a complexidade humana bem como de sua saúde, determinada por múltiplos fatores e dimensões da vida e ambiente. (PEREIRA 2000; CANDEIAS, 1997).

\section{CONCLUSÃO}

Concluimos que o estilo de vida relacionado a saúde e suas relações com a função cognitivas em idosos com vistas na promoção da saúde e suas intersecoes interdisciplinares podem possibilitar novas alternativas ou caminhos para fazer frente as demandas provocadas pelas recentes mas inexoráveis transições sociais, demográficas e epidemilogicas. Um entrelace de espaços, situações e mesmo saberes num rico, promissor e frutífero dialogo, a repercutir de modo positivo na qualidade de vida da pessoa idosa. 


\section{REFERÊNCIAS}

ABDALA, G. A.; KIMURA, M.; DUARTE, Y. C. A. et al. Religiosidade e Qualidade de Vida Relacionada à Saúde de Idosos: estudo populacional na cidade de São Paulo. Revista Saúde Pública, v. 49, p. 55, 2015.

ABDALA, G. A., et al. Religiosidade e hipertensão: estudo intervencional. Revista Formadores: vivências e estudos, v. 4, n. I, p. 33-42, 2011.

ALVES, L. C.; LEITE, I. C.; MACHADO, C. J. Conceituando e mensurando a incapacidade funcional da população idosa: uma revisão de literatura. Ciência \& Saúde Coletiva, v. 13, n. 4, p. 1199-1207, 2008.

ANTUNES, H. K. M.; SANTOS, R. F.; CASSILHAS, R.; SANTOS, R. V. T.; BUENO, O. F. A.; MELLO, M. T. Exercício físico e função cognitiva: uma revisão. Revista Brasileira de medicina do esporte, v. I2, n. 2, p. I08-114, 2006.

BRASIL. Ministério da Saúde. Secretaria de Vigilância em Saúde. Secretaria de Atenção à Saúde. Política Nacional de Promoção da Saúde. Brasília; 2006. (Série B. Textos Básicos de Saúde).

BRASIL. Ministério da Saúde. Secretaria de Atenção à Saúde. Departamento de Atenção Básica. Hipertensão arterial sistêmica para o Sistema Único de Saúde. Brasília: Cadernos de Atenção Básica. n,I5, p. 8-51, 2006.

CAMARA, F. M.; GEREZ, A. G.; MIRANDA, M. L. J.; VELARDI, M. Capacidade funcional do idoso: formas de avaliação e tendências. Acta fisiátrica, v. 15, n. 4, p. 249-262, 2008.

CANDEIAS, N. M. F. Conceitos de educação e de promoção em saúde: mudanças individuais e mudanças organizacionais. Revista de Saúde Pública v. 31, p. 209-213, 1997.

CHODZKO-ZAJKO, W.J.; MOORE, K. A. Physical fitness and cognitive functioning in aging. Exercise and sport sciences reviews, v. 22, n. I, p. 195-220, 1994.

DIAS, A. M.; CUNHA, M.; SANTOS, A. M. M.; NEVES, A. P. G.; PINTO, A. F. C.; SILVA, A. S. A., CASTRO, S. A. Adesão ao regime Terapêutico na Doença Crónica: Revisão da Literatura. Millenium-Journal of Education, Technologies, and Health, n. 40, p. 20I-219-20I-219, 2016.

FIGUEIREDO, V. L. M. Estilo de vida como indicador de saúde na velhice. Ciências \& Cognição, v. 12, p. 156-164, 2007. 
IBGE. Instituto Brasileiro de Geografia e Estatistica. SIS. Sintese de Indicadores Sociais: uma analise das condições de vida da população brasileira. Rio de Janeiro: IBGE, 2010.

KALACHE, A. O mundo envelhece: é imperativo criar um pacto de solidariedade social. Ciência \& Saúde Coletiva. v. I3, n. 4, p. IIO7-IIII, 2008.

LIMA, A. M. M.; SILVA, H. S.; GALHARDONI, R. Envelhecimento bem-sucedido: trajetórias de um constructo e novas fronteiras. Interface - Comunicação, Saúde, Educação., v.12, n.27, p.795-807, 2008.

LIRA, M.; SANTOS, L. C. C. S. Correlação entre função cognitiva e capacidade funcional nos indivíduos com doença de alzheimer. Cadernos de Pós-Graduação em Distúrbios do Desenvolvimento, São Paulo, v.12, n.2, p. 36-45, 2012.

MIRANDA, M. K. V.; PORTO, E. F.; SOUZA, A. C. Influência do estilo de vida e qualidade de vida nos exames de sangue. Temas em Saúde, v. I8, n. I, p. I43-170, 2018.

MIRANDA, R. D.; PERROTTI, T. C.; BELliNAZZI, V. R.; NÓBREGA, T. M.; CENDOROGLO, M. S.; TONIOLO, J. N. Hipertensão arterial no idoso: peculiaridades na fisiopatologia, no diagnóstico e no tratamento. Revista Brasileira Hipertensão, v. 9, n. 3, p. p. 293-300, 2002.

NASRI, F. O Envenhecimento populacional no Brasil. Einstein, . 6, n. Supl I, p. S4-S6, 2008.

OLIVEIRA, D. L. A 'nova' saúde pública e a promoção da saúde via educação: entre a tradição e a inovação. Rev Latino-americana de Enfermagem, v. 13, n. 3, p. 423-431, 2005.

PEREIRA, I. M. T.; PENTEADO, R. Z.; MARCELO, V. C. Promoção da Saúde e Educação em Saúde: uma parceria saudável. O mundo da saúde, v. 24, n. I, 2000.

RABELO, D. F. Comprometimento Cognitivo Leve em Idosos:avaliação, fatores associados e possibilidades de intervenção. Revista Kairós Gerontologia, v. I2, n. 2, p. 65-79, 2009.

RABELO, D. F.; NERI, A. L. Recursos psicológicos e ajustamento pessoal frente à incapacidade funcional na velhice. Psicologia em Estudo, Maringá, v. Io, n. 3, p. 403-412, 2005.

TEIXEIRA, E. R; LAMAS, A. R.; SILVA, J. C.; MATOS, R. M. O estilo de vida do cliente com hipertensão arterial e o cuidado com a saúde. Escola Anna Nery Revista de Enfermagem, v. Io, n. 3, p. 378-84, 2006.

VAllE, E. A.; CASTRO-COSTA, E.; FIRMO, J. O. A.; UCHOA, E.; LIMA-COSTA, M. F. Estudo de base populacional dos fatores associados ao desempenho no Mini Exame do 
Estado Mental entre idosos: Projeto Bambuí. Cadernos de Saúde Pública, v. 25, p. 918-926, 2009.

VERAS, R. P.; CALDAS, C. P.; ARAUJO, D. V;MENDES, R. K. W. A assistência suplementar de saúde e seus projetos de cuidado para com o idoso. Ciência \& saúde coletiva, v. 13, n. 4, p. III9-II26, 2008.

VERAS, R. Envelhecimento populacional contemporâneo: demandas, desafios e inovações. Revista de Saúde Pública, v. 43, n. 3, p. 548-554, 2009.

VITORINO, S. S. MIRANDA, M. L. J.; WITTER, C. Educação e envelhecimento bemsucedido: reflexões sobre saúde e autocuidado. Revista Kairós Gerontologia, v.15, n. 3, p. 2942. 2012. 